International Mathematical Forum, 2, 2007, no. 9, 441 - 453

\title{
Cost Efficiency and Cost Malmquist Productivity Index with Interval Data
}

\author{
F. Hosseinzadeh $\operatorname{Lotfi}^{1}$ \\ Dept. of Math., Science \& Research Branch \\ Islamic Azad University, Tehran, Iran \\ G. R. Jahanshahloo \\ Dept. of Math., Science \& Research Branch \\ Islamic Azad University, Tehran, Iran \\ R. Shahverdi \\ Dept. of Math., Science \& Research Branch \\ Islamic Azad University, Tehran, Iran \\ M. Rostamy-Malkhalifeh \\ Dept. of Math., Science \& Research Branch \\ Islamic Azad University, Tehran, Iran
}

\begin{abstract}
In this paper Malmquist productivity index(MPI) for decision making unit(DMU) with interval data has been evaluated.A method for assessing Malmquist productivity index using cost efficiency also has been developed.The applicability of the methods developed is illustrated in the context of the analysis of groups of teaching of university performance.
\end{abstract}

Keywords: Data Envelopment Analysis, Cost Malmquist Productivity Index, Cost Efficiency, Interval Data

Mathematics Subject Classification: 90

\footnotetext{
${ }^{1}$ Corresponding author: Hosseinzadeh_Lotfi@yahoo.com Tel, Fax:+98-21-44804172, P.O.Box:14155/775 and 14155/4933, Post Code:1477893855
} 


\section{Introduction}

Cost efficiency (CE) evaluates the ability to produce specific outputs with minimal cost. The concept of cost efficiency can be traced back to Farrell(1957) who originated many of the ideas underlying Data Envelopment Analysis(DEA). Fare et al.(1992,1994a) developed a DEA-based Malmquist productivity index which measures the productivity change over time. The Malmquist index was first suggested by Malmquist(1953) as a quantity index for use in the analysis of consumption of inputs,Fare et al. combined ideas on the measurement of efficiency from Farrell and the measurement of productivity from Caves et al.(1982) to construct a Malmquist productivity index has proven itself to be a good tool for measuring the productivity change of DMUs.

In this paper,we suggest a Malmquist productivity index measure using cost efficiency for DMUs productivity evaluation with interval data.

\section{Basic DEA Efficiency}

The underpinnings of efficiency measurement date back to the work of Debreu(1951) and Koopmans(1957). Debreu provided the first measure of efficiency, which was called the 'coefficient of resource utilization' and Koopmans was the first to define the concept of technical efficiency. Farrell (1957) extended their work in a seminal paper whose key developments were to show how to bring data to bear on Debreu's formulation of 'coefficient of resource utilization' and, looking beyond technical efficiency to propose the measurement of cost efficiency by taking into account the economic cotext in which the DMU's activity occurs. The standard DEA model that measures technical efficiency assuming costant returns to scale (CRS) and adopting an input minimisation perspective is formulated as follows(Charnes et al.,1978):

DAE input oriented model

$$
\begin{array}{lll}
\max & \theta=\sum_{r=1}^{s} u_{r} y_{r o} & (1) \\
\text { s.t. } & \sum_{i=1}^{m} v_{i} x_{i o}=1 & \\
& \sum_{r=1}^{s} u_{r} y_{r j}-\sum_{i=1}^{m} v_{i} x_{i j} \leq 0 & j=1, \ldots, n, \\
& u_{r} \geq \varepsilon & r=1, \ldots, s, \\
& v_{i} \geq \varepsilon & i=1, \ldots, m .
\end{array}
$$

In the formulation above, $\theta$ is the efficiency level of $D M U_{o}$ under assessment. $x_{i j}$ and $y_{r j}$ represent the levels of input $i$ and output $r$ at $D M U_{j} . u_{r}$ and $v_{i}$ are the weights attached to the inputs and outputs, respectively, and those are the variables of the model. $\varepsilon$ is a non-Archimedian infinitesimal use to ensure 
that all inputs and outputs are taken into account for efficiency assessment.

\section{Cost Efficiency}

Cost efficiency (CE) evaluates the ability to produce current outputs at minimal cost. In this section, we assume that prices are fixed and known, although they may possibly be different between the DMUs. In order to obtain a measure of cost efficiency for DMUs with multiple inputs and outputs, the minimum cost for the production of a DMU's current outputs with existing input prices is obtained solving the following linear problem:

minimal cost model

$$
\begin{array}{lll}
\min & \sum_{i=1}^{m} p_{i o} x_{i}^{o} & \\
\text { s.t. } & \sum_{j=1}^{n} \lambda_{j} x_{i j}=x_{i o} & i=1, \ldots, m, \\
& \sum_{j=1}^{n} \lambda_{j} y_{r j} \geq y_{r o} & r=1, \ldots, s, \\
& \lambda_{j} \geq 0 & j=1, \ldots, n, \\
& x_{i}^{o} \geq 0 & i=1, \ldots, m .
\end{array}
$$

In the model above,$p_{i o}$ is the price of input $\mathrm{i}$ for the $D M U_{o}$ under assessment. $x_{i}^{o}$ is a variable that, at the optimal solution, gives the amount of input $i$ to be used by $D M U_{o}$ in order to produce the current outputs at minimal cost. Note that,this model assumes that the input prices at each $D M U\left(p_{i o}, i=1, \ldots, m\right)$ are fixed and known,although they can differ between DMUs.

cost efficiency is then obtained as the ratio of minimum cost with specific prices (the optimal solution to model (2)) to the observed cost at $D M U_{o}$, as follows:

CostEfficiency $=\frac{\sum_{i=1}^{m} p_{i o} x_{i}^{o^{*}}}{\sum_{i=1}^{m} p_{i o} x_{i o}}$

\section{Cost Efficiency With Interval Data}

Assume that, We have $n$ DMUs with interval inputs and outputs, and $p_{i j}$ are prices input i of $D M U_{j}$ which $p_{i j} \in\left[p_{i j}^{\min }, p_{i j}^{\max }\right]$. In order to obtain a measure of cost efficiency which is an interval for this kind of data the following are suggested: 


$$
\begin{array}{lll}
\min & \sum_{i=1}^{m} p_{i o}^{\max } x_{i}^{o} & \\
\text { s.t. } & \sum_{j=1}^{n} \lambda_{j} x_{i j}^{u}=x_{i}^{o} & i=1, \ldots, m \\
& \sum_{j=1, j \neq o}^{n} \lambda_{j} y_{r j}^{l}+\lambda_{o} y_{r o}^{u} \geq y_{r o}^{u} & r=1, \ldots, s \\
& \lambda_{j} \geq 0 & j=1, \ldots, n \\
& x_{i}^{o} \geq 0 & i=1, \ldots, m .
\end{array}
$$

$$
\begin{array}{lll}
\min & \sum_{i=1}^{m} p_{i o}^{\min } x_{i}^{o} & \\
\text { s.t. } & \sum_{j=1}^{n} \lambda_{j} x_{i j}^{l}=x_{i}^{o} & i=1, \ldots, m, \\
& \sum_{j=1, j \neq o}^{n} \lambda_{j} y_{r j}^{u}+\lambda_{o} y_{r o}^{l} \geq y_{r o}^{l} & r=1, \ldots, s, \\
& \lambda_{j} \geq 0 & j=1, \ldots, n \\
& x_{i}^{o} \geq 0 & i=1, \ldots, m .
\end{array}
$$

suppose $\bar{x}_{i}^{o^{*}}$ and $\underline{x}_{i}^{o^{*}}$, are the optimal solutions for (4),(5)respectively. The following costs for $\bar{D} M U_{o}$ may be calculated as follows:

$$
\bar{c}=\frac{\sum_{i=1}^{m} p_{i o}^{\max } \bar{x}_{i}^{o^{*}}}{\sum_{i=1}^{m} p_{i o}^{\min } x_{i o}} \quad \text { (6) } \quad \underline{c}=\frac{\sum_{i=1}^{m} p_{i o}^{\min } \underline{x}_{i}^{0^{*}}}{\sum_{i=1}^{m} p_{i o}^{m a x} x_{i o}}
$$

Theorem 1): Any $c \in[\underline{c}, \bar{c}]$, can be treated as cost efficiency for $D M U_{o}$. proof: It is evident that $p_{i o} \leq p_{i o}^{\max }$. From model(4):

$x_{i}^{o^{*}}=\sum_{j=1}^{n} \lambda_{j} x_{i j}^{*} \leq \sum_{j=1}^{n} \lambda_{j} x_{i j}^{*^{u}}=x_{i}^{0^{*^{u}}}$,

And then $\sum_{i=1}^{m} p_{i o} x_{i}^{o^{*}} \leq \sum_{i=1}^{m} p_{i o}^{\max } \bar{x}_{i}^{o^{*}}(a)$. From $p_{i o} \geq p_{i o}^{\text {min }}$ we have: $\sum_{i=1}^{m} p_{i o} x_{i o} \geq \sum_{i=1}^{m} p_{i o}^{\text {min }} x_{i o}(b)$.

From (a) and (b),we obtain $c \leq \bar{c}$.In a similar way,we can show $c \geq \underline{c}$. This completes the proof.

\section{Malmquist productivity index}

By applying DEA,the best efficiency frontier will be calculated with a set of DMUs and omitting of any priority for inputs and outputs. The DMUs of efficiency frontier are the units with the maximum output and/or the minimum input levels. Using the efficient units and efficiency frontier, is the analysis of other inefficiency units possible. Malmquist productivity Index is defined with assimilation efficiency changes of each unit and technology changes. MPI can be calculated via several functions, such as distance function:

$$
D\left(X_{o}, Y_{o}\right)=\inf \left\{\theta:\left(\theta X_{o}, Y_{o}\right) \in P P S\right\}
$$

This equation shows in special conditions, only the efficiency frontier change at time $t+1$ related to $t$; that could not be a suitable criterion to calculate the technology change. This distance function does not define the inefficiency val- 
ues. The efficiency frontier will be specified for each DMU with DEA.Production function is hypothesized instant $\mathrm{t}$ and $\mathrm{t}+1$.

Calculation of the MPI needs to four linear programming problems as below: $o \in Q=\{1, \ldots, n\}$

$$
\begin{array}{lll}
D_{o}^{t}\left(X_{o}^{t}, Y_{o}^{t}\right)=\min & \theta \\
\text { s.t. } & \sum_{j=1}^{n} \lambda_{j} x_{i j}^{t} \leq \theta x_{i o}^{t} \quad & i=1, \ldots, m, \\
& \sum_{j=1}^{n} \lambda_{j} y_{r j}^{t} \geq y_{r o}^{t} \quad r=1, \ldots, s, \\
& \lambda_{j} \geq 0 & j=1, \ldots, n .
\end{array}
$$

In the model above,$x_{i o}^{t}$ is the $i^{\text {th }}$ input and $y_{r o}^{t}$ is the $r^{\text {th }}$ output of $D M U_{o}$ at time t,the value of efficiency $\left(D_{o}^{t}\left(X_{o}^{t}, Y_{o}^{t}\right)=\theta^{*}\right)$ shows that how much can be decrease inputs of $D M U_{o}$ to production that output.

If in the previous model,we put $t+1$ instead $t$, then CCR problem (9) is calculated at time t+1 and is equal $D^{t+1}\left(X_{o}^{t+1}, Y_{o}^{t+1}\right)$ and is the technical efficiency for $D M U_{o}$ at time $\mathrm{t}+1$. The value of $D^{t}\left(X_{o}^{t+1}, Y_{o}^{t+1}\right)$ for $D M U_{o}$, is the distance of $D M U_{o}$ at $\mathrm{t}+1$ with the frontier of time t,calculated by below problem:

$$
\begin{array}{lll}
D^{t}\left(X_{o}^{t+1}, Y_{o}^{t+1}\right)=\min & \theta \\
\text { s.t. } & \sum_{j=1}^{n} \lambda_{j} x_{i j}^{t} \leq \theta x_{i o}^{t+1} & i=1, \ldots, m, \\
& \sum_{j=1}^{n} \lambda_{j} y_{r j}^{t} \geq y_{r o}^{t+1} & r=1, \ldots, s, \\
& \lambda_{j} \geq 0 & j=1, \ldots, n .
\end{array}
$$

The same models $D^{t+1}\left(X_{o}^{t}, Y_{o}^{t}\right)$ and $D^{t+1}\left(X_{o}^{t+1}, Y_{o}^{t+1}\right)$ are calculated.

Fare hypotheses $D_{o}^{t+1}\left(X_{o}^{t+1}, Y_{o}^{t+1}\right)$ and $D_{o}^{t}\left(X_{o}^{t}, Y_{o}^{t}\right)$ must be equal to 1 to be efficient. Therefore he defined relative efficiency change as:

$T E C_{o}=\frac{D_{o}^{t+1}\left(X_{o}^{t+1}, Y_{o}^{t+1}\right)}{D_{o}^{t}\left(X_{o}^{t}, Y_{o}^{t}\right)}$

He described one geometric compotation to determine technology change between $\mathrm{t}$ and $\mathrm{t}+1$ :

$F S_{o}=\sqrt{\frac{D_{o}^{t}\left(X_{o}^{t+1}, Y_{o}^{t+1}\right)}{D_{o}^{t+1}\left(X_{o}^{t+1}, Y_{o}^{t+1}\right)} \cdot \frac{D_{o}^{t}\left(X_{o}^{t}, Y_{o}^{t}\right)}{D_{o}^{t+1}\left(X_{o}^{t}, Y_{o}^{t}\right)}}$

MPI will be evaluated from multiplication efficiency change and technology change for each input oriented $D M U_{o}$ at time $\mathrm{t}$ and $\mathrm{t}+1$ :

$M_{o}=\frac{D_{o}^{t+1}\left(X_{o}^{t+1}, Y_{o}^{t+1}\right)}{D_{o}^{t}\left(X_{o}^{t}, Y_{o}^{t}\right)} \cdot \sqrt{\frac{D_{o}^{t}\left(X_{o}^{t+1}, Y_{o}^{t+1}\right)}{D_{o}^{t+1}\left(X_{o}^{t+1}, Y_{o}^{t+1}\right)} \cdot \frac{D_{o}^{t}\left(X_{o}^{t}, Y_{o}^{t}\right)}{D_{o}^{t+1}\left(X_{o}^{t}, Y_{o}^{t}\right)}}$ 
The simple form of relation (13)is:

$M_{o}=\sqrt{\frac{D_{o}^{t}\left(X_{o}^{t+1}, Y_{o}^{t+1}\right)}{D_{o}^{t}\left(X_{o}^{t}, Y_{o}^{t}\right)} \cdot \frac{D_{o}^{t+1}\left(X_{o}^{t+1}, Y_{o}^{t+1}\right)}{D_{o}^{t+1}\left(X_{o}^{t}, Y_{o}^{t}\right)}}$

So,we have three conditions:

1. $M_{o}>1$,increase productivity and observe progress.

$2 . M_{o}<1$, decrease productivity and observe regress.

$3 . M_{o}=1$,no change in productivity at time $\mathrm{t}+1$ in comparison to $\mathrm{t}$.

\section{Cost Malmquist productivity index}

\subsection{Background}

Consider that in time period t, producers are using inputs $x^{t} \in R^{m}$, to produce outputs $y^{t} \in R^{s}$. Define now the production technology of period $\mathrm{t}$ in terms of the input requirement set, which is:

$L^{t}\left(y^{t}\right)=\left\{x^{t}: x^{t}\right.$ can produce $\left.y^{t}\right\}$

$L^{t}\left(y^{t}\right)$ contains all input vectors that can produce output $y^{t}$. Assume that $L^{t}\left(y^{t}\right)$ is non-empty, closed, convex, bounded and satisfies strong disposability of inputs and outputs. $L^{t}\left(y^{t}\right)$ is bounded from below by the input isoquant, that is:

$\operatorname{Isoq} L^{t}\left(y^{t}\right)=\left\{x^{t}: x^{t} \in L^{t}\left(y^{t}\right), \lambda x^{t} \notin L^{t}\left(y^{t}\right)\right.$ for $\left.\lambda<1\right\}$

Isoq $L^{t}\left(y^{t}\right)$ defines a boundary (frontier) to the input requirement set in the sense that any radial contraction of input vectors that lie on the frontier is not possible within $L^{t}\left(y^{t}\right)$. Alternatively, with reference to the input requirement set, define the technology of production in terms of the input distance function as:

$D_{i}^{t}\left(x^{t}, y^{t}\right)=\sup \left\{\theta:\left(\frac{x^{t}}{\theta}\right) \in L^{t}\left(y^{t}\right), \theta>0\right\}$

where the subscript i denotes input orientation.

$D_{i}^{t}\left(x^{t}, y^{t}\right)$ in (17) is the largest factor by which the input levels in $x^{t}$ can be divided while $x^{t}$ remains in $L^{t}\left(y^{t}\right)$. If $D_{i}^{t}\left(x^{t}, y^{t}\right) \geq 1$, then it is sufficient for $x^{t} \in L^{t}\left(y^{t}\right)$ and if $D_{i}^{t}\left(x^{t}, y^{t}\right)=1$ then $x^{t} \in I \operatorname{soq} L^{t}\left(y^{t}\right) . D_{i}^{t}\left(x^{t}, y^{t}\right)$ is similar with the definition of technical efficiency in input oriented: 
$T E_{i}^{t}\left(x^{t}, y^{t}\right)=\min \left\{\phi: \phi x^{t} \in L^{t}\left(y^{t}\right), \phi>0\right\}$

When input prices, $P^{t} \in R^{m}$, are available, the cost function is defined:

$C^{t}\left(y^{t}, p^{t}\right)=\min \left\{p^{t} x^{t}: x^{t} \in L^{t}\left(y^{t}\right), p^{t}>0\right\}$

$C^{t}\left(y^{t}, p^{t}\right)$ is the minimum cost of producing a given output vector $y^{t}$ given the input prices $p^{t}$ and the technology of period t. The set of input vectors $x^{t}$ which correspond to the scalar $C^{t}\left(y^{t}, p^{t}\right)$ lie on an isocost line which defines a cost boundary ??(versus production boundary)??defined as:

$I s o \bar{C}^{t}\left(y^{t}, p^{t}\right)=\left\{x^{t}: p^{t} x^{t}=C^{t}\left(y^{t}, p^{t}\right)\right\}$

This boundary contains the input vectors that can have the minimum cost with their price $p^{t}$.Therfore technical efficiency and distance function have the same definition. Overall(or cost)efficiency defines:

$O E_{i}^{t}\left(y^{t}, x^{t}, p^{t}\right)=\frac{C^{t}\left(y^{t}, p^{t}\right)}{p^{t} x^{t}}$

because technical efficiency is less than overall efficiency for each unit ,then:

$T E_{i}^{t}\left(x^{t}, y^{t}\right) \leq O E_{i}^{t}\left(y^{t}, x^{t}, p^{t}\right)$

According to technical efficiency is the same as distance function:

$C^{t}\left(y^{t}, p^{t}\right) \leq \frac{p^{t} x^{t}}{D_{i}^{t}\left(y^{t}, x^{t}\right)}$

Allocative efficiency defines as follows:

$A E_{i}^{t}\left(y^{t}, x^{t}, p^{t}\right)=\frac{C^{t}\left(y^{t}, p^{t}\right) D_{i}^{t}\left(y^{t}, x^{t}\right)}{p^{t} x^{t}}$

Assume two time periods $\mathrm{t}$ and $\mathrm{t}+1$ respectively and define in each one of them technology and production. Taking time period $t$ as the reference period, the input oriented Malmquist index (IM) is:

$I M^{t}=\frac{D_{i}^{t}\left(y^{t+1}, x^{t+1}\right)}{D_{i}^{t}\left(y^{t}, x^{t}\right)}$

In a similar fashion, with reference to period $t+1$, one may define the following index:

$I M^{t+1}=\frac{D_{i}^{t+1}\left(y^{t+1}, x^{t+1}\right)}{D_{i}^{t+1}\left(y^{t}, x^{t}\right)}$

To avoid an arbitrary choice of a reference period Fare et al. use the geometric 
mean of IM $t$ and IM $t+1$ so that the IM is:

$I M=\sqrt{\frac{D_{i}^{t}\left(y^{t+1}, x^{t+1}\right)}{D_{i}^{t}\left(y^{t}, x^{t}\right)} \frac{D_{i}^{t+1}\left(y^{t+1}, x^{t+1}\right)}{D_{i}^{t+1}\left(y^{t}, x^{t}\right)}}$

IM is Malmquist productivity index and has inverse relative with $M_{o}$ definition previous section. Three conditions are existed:

1.I $M<1$, observe progress.

$2 . I M>1$, observe regress.

$3 . I M=1$, do not observe any change in productivity .

\subsection{Cost Malmquist productivity index}

By using allocative and technical efficiency, input's price productivity changes are determined.Specifically, in the spirit of the indexes in (25)-(27) define a dual cost Malmquist $(\mathrm{CM})$ productivity index of period $t, t+1$ and their geometric mean as follows:

$C M^{t}=\left[\frac{p^{t} x^{t+1} / C^{t}\left(y^{t+1}, p^{t}\right)}{p^{t} x^{t} / C^{t}\left(y^{t}, p^{t}\right)}\right]$

$C M^{t+1}=\left[\frac{p^{t+1} x^{t+1} / C^{t+1}\left(y^{t+1}+1, p^{t+1}\right)}{p^{t+1} x^{t} / C^{t+1}\left(y^{t}, p^{t+1}\right)}\right]$

$C M=\left[\frac{p^{t} x^{t+1} / C^{t}\left(y^{t+1}, p^{t}\right)}{p^{t} x^{t} / C^{t}\left(y^{t}, p^{t}\right)} \frac{p^{t+1} x^{t+1} / C^{t+1}\left(y^{t+1}, p^{t+1}\right)}{p^{t+1} x^{t} / C^{t+1}\left(y^{t}, p^{t+1}\right)}\right]^{\frac{1}{2}}$

where $p^{t} x^{t}=\sum_{n=1}^{N} p_{n}^{t} x_{n}^{t}$, n denotes the nth input and $C^{t}\left(y^{t}, p^{t}\right)$ is as defined in (19) with reference to the CRS technology.

The cost ratio $p^{t} x^{t} / C^{t}\left(y^{t}, p^{t}\right)$ measures the extent to which the aggregate production cost in period t can be reduced while still securing the output vector $y^{t}$ under the input price vector $p^{t}$. This ratio measures the distance between the observed cost $p^{t} x^{t}$ and the cost boundary $C^{t}\left(y^{t}, p^{t}\right)$. This distance will have a minimum value of 1 , (when the two costs coincide) and the larger it is the larger the factor by which the observed cost of securing output $y^{t}$ can be reduced.This (cost) distance is the reciprocal of the input oriented measure of overall efficiency defined in (21). The rest of the cost ratios in (28)-(30) are defined in an analogous manner.

Thus, the CM index is defined in terms of distances by which input costs can be deflated (inflated) to reach a cost boundary. This is in contrast to the IM index which is defined in terms of distances by which input quantities can be deflated( inflated) to reach a production boundary. Note at this point that again we do not need to make assumptions about the returns to scale that characterize the technology. We use the CRS cost boundaries as benchmarks for productivity measurement. Just as with the IM index, a CM index value less than 1 implies productivity progress, a value greater than 1 implies regress 
and a value of 1 indicates constant productivity.

\section{$7 \quad$ Malmquist Productivity Index With Inter- val Data}

In the section (4), we defined Malmquist productivity index. With interval data the Malmquist productivity index may be defined as follows.Consider four models in the following form,so the M.P.I is an interval which is defined.

$$
\begin{aligned}
& \underline{\theta}_{t}^{t}=\min \theta \\
& \text { s.t. } \quad \sum_{j=1}^{n} \lambda_{j} x_{i j}^{t l} \leq \theta x_{i o}^{t u} \quad i=1, \ldots, m, \\
& \begin{array}{ll}
\sum_{j=1}^{n} \lambda_{j} y_{r j}^{t u} \geq y_{r o}^{t l} & r=1, \ldots, s, \\
\lambda_{j} \geq 0 & j=1, \ldots, n .
\end{array} \\
& \bar{\theta}_{t}^{t+1}=\min \theta \\
& \text { s.t. } \quad \sum_{j=1}^{n} \lambda_{j} x_{i j}^{t u} \leq \theta x_{i o}^{t+1 l} \quad i=1, \ldots, m \text {, } \\
& \sum_{j=1}^{n} \lambda_{j} y_{r j}^{t l} \geq y_{\text {ro }}^{t+1 u} \quad r=1, \ldots, s, \\
& \lambda_{j} \geq 0 \quad j=1, \ldots, n \text {. } \\
& \bar{\theta}_{t+1}^{t+1}=\min \theta \\
& \text { s.t. } \quad \sum_{j=1}^{n} \lambda_{j} x_{i j}^{t+1 u} \leq \theta x_{i o}^{t+1 l} \quad i=1, \ldots, m \text {, } \\
& \sum_{j=1}^{n} \lambda_{j} y_{r j}^{t+1 l} \geq y_{\text {ro }}^{t+1 u} \quad r=1, \ldots, s, \\
& \lambda_{j} \geq 0 \quad j=1, \ldots, n \text {. } \\
& \underline{\theta}_{t+1}^{t}=\min \theta \\
& \text { s.t. } \quad \sum_{j=1}^{n} \lambda_{j} x_{i j}^{t+1 l} \leq \theta x_{i o}^{t u} \quad i=1, \ldots, m, \\
& \sum_{j=1}^{n} \lambda_{j} y_{r j}^{t+1 u} \geq y_{r o}^{t l} \quad r=1, \ldots, s, \\
& \lambda_{j} \geq 0 \quad j=1, \ldots, n \text {. }
\end{aligned}
$$

Therefore from the above models, we have: $\bar{M}=\left[\frac{\bar{\theta}_{t}^{t+1}}{\underline{\underline{\theta}}_{t}^{t}} \times \frac{\bar{\theta}_{t+1}^{t+1}}{\underline{\theta}_{t+1}^{t}}\right]^{\frac{1}{2}}$

The values $\bar{\theta}_{t}^{t}, \bar{\theta}_{t+1}^{t}, \underline{\theta}_{t}^{t+1}$ and $\underline{\theta}_{t+1}^{t+1}$ can be evaluated,too.So,we obtain:

$\underline{M}=\left[\frac{\underline{\theta}_{t}^{t+1}}{\bar{\theta}_{t}^{t}} \times \frac{\underline{\theta}_{t+1}^{t+1}}{\bar{\theta}_{t+1}^{t}}\right]^{\frac{1}{2}}$

Theorem 2):By using of previous models $M \in[\underline{M}, \bar{M}]$, that $\mathrm{M}$ is the Malmquist productivity index.

proof: Proof is evident. 


\section{Cost Malmquist with Interval Data}

In the above mentioned model,it has been assumed that $p_{i j}$ were fixed.If $p_{i j}$ lies in the interval,the following models may be used to evaluate the M.P.I.

$$
\begin{array}{lll}
\min & \sum_{i=1}^{m} p_{i o}^{\max } x_{i}^{o} & (37) \\
\text { s.t. } & \sum_{j=1}^{n} \lambda_{j} x_{i j}^{t+1 u}=x_{i}^{o} & i=1, \ldots, m \\
& \sum_{j=1, j \neq o}^{n} \lambda_{j} y_{r j}^{t+1 l}+\lambda_{o} y_{r o}^{t+1 u} \geq y_{r o}^{t+1 u} & r=1, \ldots, s \\
& \lambda_{j} \geq 0 & j=1, \ldots, n \\
& x_{i}^{o} \geq 0 & i=1, \ldots, m .
\end{array}
$$

$$
\begin{array}{lll}
\min & \sum_{i=1}^{m} p_{i o}^{\max } x_{i}^{o} & \\
\text { s.t. } & \sum_{j=1}^{n} \lambda_{j} x_{i j}^{t u}=x_{i}^{o} & i=1, \ldots, m \\
& \sum_{j=1}^{n} \lambda_{j} y_{r j}^{t l} \geq y_{r o}^{t+1 u} & r=1, \ldots, s \\
& \lambda_{j} \geq 0 & j=1, \ldots, n \\
& x_{i}^{o} \geq 0 & i=1, \ldots, m .
\end{array}
$$

$$
\begin{array}{llr}
\min & \sum_{i=1}^{m} p_{i o}^{m i n} x_{i}^{o} & (39) \\
\text { s.t. } & \sum_{j=1}^{n} \lambda_{j} x_{i j}^{t l}=x_{i}^{o} & i=1, \ldots, m \\
& \sum_{j=1, j \neq o}^{n} \lambda_{j} y_{r j}^{t u}+\lambda_{o} y_{r o}^{t l} \geq y_{r o}^{t l} & r=1, \ldots, s, \\
& \lambda_{j} \geq 0 & j=1, \ldots, n \\
& x_{i}^{o} \geq 0 & i=1, \ldots, m .
\end{array}
$$

$$
\begin{array}{lll}
\min & \sum_{i=1}^{m} p_{i o}^{m i n} x_{i}^{o} \\
\text { s.t. } & \sum_{j=1}^{n} \lambda_{j} x_{i j}^{t+1 l}=x_{i}^{o} & i=1, \ldots, m \\
& \sum_{j=1}^{n} \lambda_{j} y_{r j}^{t+1 u} \geq y_{r o}^{t l} & r=1, \ldots, s \\
& \lambda_{j} \geq 0 & j=1, \ldots, n \\
& x_{i}^{o} \geq 0 & i=1, \ldots, m .
\end{array}
$$

The following costs from models (37),(38),(39)and(40) may be calculated as follows, respectively:

$$
\begin{aligned}
\bar{c}_{t+1}^{t+1} & =\frac{\sum_{i=1}^{m} p_{i o}^{\max } \bar{x}_{i}^{o^{*}}}{\sum_{i=1}^{m} p_{i o}^{m i n} x_{i o}}, & \bar{c}_{t}^{t+1} & =\frac{\sum_{i=1}^{m} p_{i o}^{m a x} \bar{x}_{i}^{o^{*}}}{\sum_{i=1}^{m} p_{i o}^{\min } x_{i o}} \\
\underline{c}_{t}^{t} & =\frac{\sum_{i=1}^{m} p_{i o}^{m i n} \underline{x}_{i}^{o^{*}}}{\sum_{i=1}^{m} p_{i o}^{m a x} x_{i o}}, & \underline{c}_{t+1}^{t} & =\frac{\sum_{i=1}^{m} p_{i o}^{m i n} \underline{o}_{i}^{o^{*}}}{\sum_{i=1}^{m} p_{i o}^{m a x} x_{i o}} .
\end{aligned}
$$

Therefore,we evaluate from above models $\bar{M}_{c}$ as following:

$$
\bar{M}_{c}=\left[\frac{\bar{c}_{t}^{t+1}}{\underline{c}_{t}^{t}} \times \frac{\bar{c}_{t+1}^{t+1}}{\underline{\underline{c}}_{t+1}^{t}}\right]^{\frac{1}{2}}
$$

In a similar fashion,we can obtain $\underline{M}_{c}$ with using of previous models as following:

$\underline{M}_{c}=\left[\frac{\underline{\underline{c}}_{t}^{t+1}}{\bar{c}_{t}^{t}} \times \frac{\underline{c}_{t+1}^{t+1}}{\bar{c}_{t+1}^{t}}\right]^{\frac{1}{2}}$ 
Theorem 3): Any $M_{c} \in\left[\underline{M}_{c}, \bar{M}_{c}\right]$, that $M_{c}$ is Cost Malmquist productivity index.

\section{Application Example}

In this section,we investigate Malmquist Productivity Index, when the prices of some inputs are interval.The conclusion of these observations are explained in following tables. Consider eighteen groups of teaching of

ZAHEDAN university that inputs and outputs show in the following table:

\begin{tabular}{|l|l|}
\hline \multicolumn{1}{|c|}{ Input } & Output \\
\hline 1)Number of expert & 1)Average of mean \\
2)Area of room & 2)Number of student in 2003 \\
3)Number of computer & 3)successful in high graduate \\
4)Average of mean graduate & \\
5)Mean of full grade & \\
6)Average of full rank & \\
7)Number of teacher & \\
8)Number of conditional & \\
students in 2003 & \\
9)Number of deport & \\
student in 2003 & \\
\hline
\end{tabular}

Table(1):Inputs and Outputs

We suppose that the average of salary of a expert is between 1000000 to 1500000 Rials.Each square meter of a room is 2000000 Rials.Also, the average price of a computer was in interval [3000000,7000000] and the average of salary of teachers are changed between 3000000 and 7000000 Rials.

Cost Malmquist productivity index for these eighteen groups of teaching are showing in the following table: 


\begin{tabular}{|c|c|c|}
\hline DMU & $\underline{M}_{c}$ & $\bar{M}_{c}$ \\
\hline 1 & 0.73422850 & 2.91897837 \\
2 & 0.75054921 & 2.62242136 \\
3 & 0.70758376 & 2.84616188 \\
4 & 0.42705200 & 1.08220943 \\
5 & 0.32539743 & 3.48498139 \\
6 & 2.40927830 & 12.74087508 \\
7 & 0.52388458 & 2.57570445 \\
8 & 0.41019126 & 0.59080896 \\
9 & 0.65081897 & 1.03231053 \\
10 & 1.31784815 & 3.20261519 \\
11 & 0.55077361 & 0.69243311 \\
12 & 0.59371170 & 2.26191876 \\
13 & 0.58065872 & 252002219 \\
14 & 0.72228332 & 1.60369503 \\
15 & 0.16127203 & 0.20323370 \\
16 & 1.00147195 & 1.37689241 \\
17 & 3.83455276 & 8.54141155 \\
18 & 0.52123521 & 0.51836845 \\
\hline
\end{tabular}

Table(2):Cost Malmquist

\section{0 conclusion}

In the above example, if $\underline{M}_{c}$ and $\bar{M}_{c}$ both of them are greater than one, then we will have progress and if $\underline{M}_{c}$ and $\bar{M}_{c}$ both of them are smaller than one,then we will have regress.But if $\underline{M}_{c}$ is smaller than one and $\bar{M}_{c}$ is greater than one,then we couldn't say anything.(It depends on the view of manager).This paper explored the assessment of productivity index with interval data and using cost efficiency. The applicability of the methods in bank branch seems to be very useful and consistent with common sense of board of directory and managerial port of view.It seems MI can be assessed using revenue efficiency.

\section{References}

[1]Berg,S. A.,Forsund,F. R.,Jansen,F. S.,1992.Malmquist indices of Productivity growth during the deregulation of Norwegian banking,1980-89.Scandinavian Journal of economics(supplement),211-228.

[2]Caves,D.W.,Christensen,L. R.,Diewert,W.,E.,1982.The economic theory of index numbers and the measurement of input,output and productivity. 
Econometrica.50,1393-1414.

[3]Charnes,A.,Cooper,W.W.,Rhodes,E.,1978.Measuring the efficiency of decision making units. European Journal of Operational Research 2, 429-444.

[4]Debreu,G.,1951. The coefficient of resource utilisation. Econometrica 19(3), 273-292.

[5]Despotis,D.K.,Smirlis,Y.G.,2002.Data envelopment analysis with imprecise data. European Journal of Operational Research. 140, 24-36.

[6]Dyson,R.G.,Camanho,A.S.,2005.Cost efficiency measurement with price uncertainty, a DEA application to bank branch assessments.European Journal of Operational Research.161,432-446.

[7]Fare,R.,Grosskopf,Norris M.,Zhang,Z.,1994. Productivity growth,technical progress, and efficiency change in industrialized counties. American Economic Review. 84, 66-83.

[8]Malmquist,S.,1953.Index numbers and indifference surfaces. Trabajos de Estadistica.4,209-242.

[9]Thanassoulis,E.,Maniadakis,N.,2004.A cost Malmquist productivity index. European Journal of Operational Research. 154, 396-409.

Received: November 29, 2005 